\title{
Team QCRI-MIT at SemEval-2019 Task 4: Propaganda Analysis Meets Hyperpartisan News Detection
}

\author{
Abdelrhman Saleh ${ }^{1}$, Ramy Baly ${ }^{2}$, Alberto Barrón-Cedeño ${ }^{3}$, \\ Giovanni Da San Martino ${ }^{3}$, Mitra Mohtarami ${ }^{2}$, Preslav Nakov ${ }^{3}$, James Glass ${ }^{2}$ \\ ${ }^{1}$ Harvard University, MA, USA \\ ${ }^{2}$ MIT Computer Science and Artificial Intelligence Laboratory, MA, USA \\ ${ }^{3}$ Qatar Computing Research Institute, HBKU, Qatar \\ abdelrhman_salehecollege.harvard.edu, \\ $\{$ baly, mitram, glass\}@mit.edu \\ \{albarron, gmartino, pnakov\}@hbku.edu.qa
}

\begin{abstract}
We describe our submission to SemEval-2019 Task 4 on Hyperpartisan News Detection. We rely on a variety of engineered features originally used to detect propaganda. This is based on the assumption that biased messages are propagandistic and promote a particular political cause or viewpoint. In particular, we trained a logistic regression model with features ranging from simple bag of words to vocabulary richness and text readability. Our system achieved $72.9 \%$ accuracy on the manually annotated testset, and $60.8 \%$ on the test data that was obtained with distant supervision. Additional experiments showed that significant performance gains can be achieved with better feature pre-processing. ${ }^{1}$
\end{abstract}

\section{Introduction}

The rise of social media has enabled people to easily share information with a large audience without regulations or quality control. This has allowed malicious users to spread disinformation and misinformation (a.k.a. "fake news") at an unprecedented rate. Fake news is typically characterized as being hyperpartisan (one-sided), emotional and riddled with lies (Potthast et al., 2018). The SemEval-2019 Task 4 on Hyperpartisan News Detection (Kiesel et al., 2019) focused on the challenge of automatically identifying whether a text is hyperpartisan or not.

While hyperpartisanship is defined as "exhibiting one or more of blind, prejudiced, or unreasoning allegiance to one party, faction, cause, or person", we model this task as a binary document classification problem. Scholars have argued that all biased messages can be considered propagandistic, regardless of whether the bias was intentional or not (Ellul, 1965, p. XV).

\footnotetext{
${ }^{1}$ Our system is available at https://github.com/ AbdulSaleh/QCRI-MIT-SemEval2019-Task4
}

Thus, we approached the task departing from an existing model for propaganda identification (Barrón-Cedeño et al., 2019). Our hypothesis is that propaganda is inherent in hyperpartisanship and that the two problems are two sides of the same coin, and thus solving one of them would help solve the other. Our system consists of a logistic regression model that is trained with a variety of engineered features that range from word and character TF.IDF $n$-grams and lexicon-based features to more sophisticated features that represent different aspects of the article's text such vocabulary richness and language complexity.

Our official submission achieved an accuracy of $72.9 \%$ (while the winning system achieved $82.2 \%$ ). This was achieved using word and character $n$-grams. Moreover, post-submission experiments have shown that further performance improvements can be achieved by carefully preprocessing the engineered features.

\section{Related Work}

The analysis of bias and disinformation has attracted significant attention, especially after the 2016 US presidential election (Brill, 2001; Finberg et al., 2002; Castillo et al., 2011; Baly et al., 2018a; Kulkarni et al., 2018; Mihaylov et al., 2018; Baly et al., 2019). Most approaches have focused on predicting credibility, bias or stance.

Stance detection was considered as an intermediate step for detecting fake claims, where the veracity of a claim is checked by aggregating the stances of the retrieved relevant articles (Baly et al., 2018b; Nakov et al., 2019). Several stance detection models have been proposed including deep convolutional neural networks (Baird et al., 2017), multi-layer perceptrons (Hanselowski et al., 2018), and end-to-end memory networks (Mohtarami et al., 2018). 
The stylometric analysis model of Koppel et al. (2007) was used by Potthast et al. (2018) to address hyperpartisanship. They used articles from nine news sources whose factuality has been manually verified by professional journalists. Writing style and complexity were also considered by Horne and Adal (2017) to differentiate real news from fake news and satire. They used features such as the number of occurrences of different part-of-speech tags, swearing and slang words, stop words, punctuation, and negation as stylistic markers. They also used a number of readability measures. Rashkin et al. (2017) focused on a multi-class setting (real news vs. satire vs. hoax vs. propaganda) and relied on word $n$-grams.

Similarly to Potthast et al. (2018), we believe that there is an inherent style in propaganda, regardless of the source publishing it. Many stylistic features were proposed for authorship identification, i.e., the task of predicting whether a piece of text has been written by a particular author. One of the most successful representations for such a task are character-level $n$-grams (Stamatatos, 2009), and they turn out to represent some of our most important stylistic features.

More details about research on fact-checking and the spread of fake news online can be found in recent surveys (Lazer et al., 2018; Vosoughi et al., 2018; Thorne and Vlachos, 2018).

\section{System Description}

We developed our system for detecting hyperpartisanship in news articles by training a logistic regression classifier using features such as character and word $n$-grams, lexicon-based indicators, and readability and vocabulary richness measures. Below, we describe these features in detail.

Character 3-grams. Stamatatos (2009) argued that, for tasks where the topic is irrelevant, character-level representations are more sensitive than token-level ones. We hypothesize that this applies to hyperpartisan news detection, since articles on both sides of the political spectrum may be discussing the same topics. Stamatatos (2009) found that "the most frequent character $n$-grams are the most important features for stylistic purposes". These features capture different style markers, such as prefixes, suffixes and punctuation marks. Following the analysis in Barrón-Cedeño et al. (2019), we include TF.IDF-weighted character 3-grams in our feature set.
Word $n$-grams Bag-of-words (BoW) features are widely used for text classification. We extracted the $k$ most frequent $[1,2]$-grams, and we represented them using their TF.IDF scores. We ignored $n$-grams that appeared in more than $90 \%$ of the documents, most of which contained stopwords and were irrelevant with respect to hyperpartisanship. Furthermore, we incorporated Naive Bayes by weighing the $n$-grams based on their importance for classification, as proposed by Wang and Manning (2012). We define $\boldsymbol{x}_{i} \in \mathbb{R}^{|V|}$ as a row vector in the TF.IDF feature matrix, representing the $i^{\text {th }}$ training sample with a target label $y_{i} \in\{0,1\}$, where $V$ is the vocabulary size. We also define vectors $\boldsymbol{p}=\alpha+\sum_{i: y_{i}=1} \boldsymbol{x}_{i}$ and $\boldsymbol{q}=\alpha+\sum_{i: y_{i}=0} \boldsymbol{x}_{i}$, and we set the smoothing parameter $\alpha$ to 1 . Finally, we calculate the vector:

$$
\boldsymbol{r}=\log \left(\frac{\boldsymbol{p} /\|\boldsymbol{p}\|}{\boldsymbol{q} /\|\boldsymbol{q}\|}\right)
$$

which is used to scale the TF.IDF features to create the NB-TF.IDF features as follows:

$$
\boldsymbol{x}_{i}^{\prime}=\boldsymbol{r} \circ \boldsymbol{x}_{i}, \quad \forall i
$$

Bias Analysis We analyze the bias in the language used in the documents by (i) creating bias lexicons that contain left and right bias cues, and (ii) using these lexicons to compute two scores for each document, indicating the intensity of bias towards each ideology. To generate the list of cues that signal biased language, we use Semantic Orientation (SO) (Turney, 2002) to identify the words that are strongly associated with each of the left and right documents in the training dataset. Those SO values can be either positive or negative, indicating association with right or left biases, respectively. Then, we select words whose absolute SO value is $\geq 0.4$ to create two bias lexicons: $B L_{\text {left }}$ and $B L_{\text {right }}$. Finally, we use these lexicons to compute two bias scores per document according to Equation (3), where for each document $D_{j}$, the frequency of cues in the lexicon $B L_{i}$ that are present in $D_{j}$ is normalized by the total number of words in $D_{j}$ :

$$
\operatorname{bias}_{i}\left(D_{j}\right)=\frac{\sum_{c u e \in B L_{i}} \operatorname{count}\left(\text { cue }, D_{j}\right)}{\sum_{w_{k} \in D_{j}} \operatorname{count}\left(w_{k}, D_{j}\right)}
$$


Lexicon-based Features. Rashkin et al. (2017) studied the occurrence of specific types of words in different kinds of articles, and showed that words from certain lexicons (e.g., negation and swear words) appear more frequently in propaganda, satire, and hoax articles than in trustworthy articles. We capture this by extracting features that reflect the frequency of words from particular lexicons. We use 18 lexicons from Wiktionary, Linguistic Inquiry and Word Count (LIWC) (Pennebaker et al., 2001), Wilson's subjectives (Wilson et al., 2005), Hyland's hedges (Hyland, 2015), and Hooper's assertives (Hooper, 1975). For each lexicon, we count the total number of words in the article that appear in the lexicon. This resulted in 18 features, one for each lexicon.

Vocabulary Richness Potthast et al. (2018) showed that hyperpartisan outlets tend to use a writing style that is different from mainstream outlets. Different topic-independent features have been proposed to characterize the vocabulary richness, style and complexity of a text. For this task, we used the following vocabulary richness features: (i) type-token ratio (TTR), or the ratio of types to tokens in the text, (ii) Hapax Legomena, or the number of word types appearing only once in the text, (iii) Hapax Dislegomena, or the number of types appearing twice in the text, (iv) Honore's $R$, which is calculated as a combination of types, tokens, and hapax legomena (Honore, 1979):

$$
\text { Honore's } \mathrm{R}=\frac{100 \times \log (\mid \text { tokens } \mid)}{1-\mid \text { Legomena }|/| \text { types } \mid}
$$

We further used (v) Yule's characteristic $K$, which is defined as the chance of a word occurring in a text, estimated as following a Poisson distribution (Yule, 1944):

$$
\text { Yule's K }=10^{4} \cdot \frac{\sum_{i} i^{2} \mid \text { types }_{i}|-| \text { tokens } \mid}{\mid \text { tokens }^{2}} \text {, }
$$

where tokens refer to all words in a text (including repetitions), types refer to distinct words, $i$ are the tokens' frequency ranks ( 1 being the least frequent), and types $i$ are the number of tokens with the $i^{\text {th }}$ frequency.
Readability We also used the following readability features, which were originally designed to estimate the level of text complexity: (i) FleschKincaid grade level represents the US grade level necessary to understand a text (Kincaid et al., 1975), (ii) Flesch reading ease is a score for measuring how difficult a text is to read (Kincaid et al., 1975), and (iii) Gunning fog index estimates the years of formal education necessary to understand a text (Gunning, 1968).

\section{Experiments and Results}

\subsection{Dataset}

We trained our models on the Hyperpartisan News Dataset from SemEval-2019 Task 4 (Kiesel et al., 2019), which is split by the task organizers into

(i) Labeled by-Publisher, with $750 \mathrm{~K}$ articles labeled via distant supervision, i.e., using labels for their publisher. ${ }^{2}$ The labels are evenly distributed between "hyperpartisan" and "not-hyperpartisan." This set is further split into $600 \mathrm{~K}$ articles for training and $150 \mathrm{~K}$ for validation.

(ii) Labeled by-Article: This set contains 645 articles labeled using crowd-sourcing (37\% are hyperpartisan and $63 \%$ are not). Only articles with a consensus among the annotators were included.

\subsection{Experimental Settings}

We trained a logistic regression (LR) model with a Stochastic Average Gradient solver (Schmidt et al., 2017) due to the large size of the dataset. In order to reduce overfitting, we used $\mathbb{L}_{2}$ regularization (with $C=1$ as the regularization parameter). Moreover, feature normalization was needed since the different features represent different aspects of the text, and thus have very different scales. We tried to normalize each feature set by subtracting the mean and then scaling it to unit variance. However, we found that multiplying the features by constant scaling factors resulted in better performance. The scaling factor for each family of features was a hyperparameter that we tuned on the validation dataset.

We trained the classifier using the $600 \mathrm{~K}$ training examples annotated by-Publisher, then we used the remaining $150 \mathrm{~K}$ examples for evaluation. We fine-tuned the hyperparameters on the 645 byArticle examples.

\footnotetext{
${ }^{2}$ The publisher's labels are identified by BuzzFeed journalists or by the Media Bias/Fact Check project
} 


\begin{tabular}{|c|c|c|c|c|c|c|c|c|c|}
\hline & \multirow{2}{*}{ Features } & \multicolumn{4}{|c|}{ Labeled by-Article } & \multicolumn{4}{|c|}{ Labeled by-Publisher } \\
\hline & & Accuracy & Prec. & Rec. & $\mathrm{F} 1$ & Accuracy & Prec. & Rec. & $\mathrm{F} 1$ \\
\hline 1 & BoW (TF.IDF) & 67.8 & 53.8 & 89.1 & 67.1 & 56.7 & 55.1 & 72.5 & 62.6 \\
\hline 2 & BoW (NB-TF.IDF) & 69.6 & 56.1 & 80.7 & 66.2 & 57.1 & 56.4 & 61.9 & 59.0 \\
\hline 3 & $\llcorner+$ Char trigrams & 74.0 & 62.5 & 73.5 & 67.6 & 54.8 & 54.3 & 60.8 & 57.4 \\
\hline 4 & $\llcorner+$ Bias & 75.2 & 67.7 & 62.6 & 65.1 & 54.5 & 55.0 & 50.4 & 52.6 \\
\hline 5 & ᄂ+ Lexical & 75.2 & 67.0 & 64.7 & 65.8 & 52.3 & 52.3 & 51.5 & 51.9 \\
\hline 6 & L+ Vocab. Richness & 75.8 & 67.1 & 67.6 & 67.4 & 50.9 & 50.8 & 52.5 & 51.7 \\
\hline 7 & $\llcorner+$ Readability & 76.0 & 66.4 & 70.6 & 68.4 & 51.6 & 51.5 & 53.9 & 52.7 \\
\hline
\end{tabular}

Table 1: An incremental analysis showing the performance of different feature combinations, evaluated on the validation datasets labeled by article and by publisher.

The hyper-parameters include the number of most frequent word $n$-grams $k, k \in[50,200,700]^{\times 10^{3}}$, and the scaling parameters of the features, except for the $n$-grams. Eventually, we set $k=200,000$, and we used the most-frequent word [1,2]-grams. Moreover, we assessed the different feature sets, described in Section 3 by incrementally adding each set, one at a time, to the mix of all features.

\subsection{Results}

Table 1 illustrates the results obtained on both the by-Article set (which we used to fine-tune the model's hyper-parameters) and the by-Publisher set (which we used for evaluation). Our results suggest that scaling the TF.IDF values through Naive Bayes is better than using raw TF.IDF scores. Hence, this is what we used in subsequent experiments. We can also see that adding each group of features introduces a consistent improvement in accuracy on the by-Article data. However, we observed an opposite behaviour on the byPublisher data. We believe this is due to the significant amount of noisy labels introduced by the distant supervision labeling strategy. Therefore, we based our decisions on the results obtained on the by-Article data since its labels are more accurate.

The normalization strategy, i.e., scaling the features using calibrated scaling parameters, yielded significant performance improvements. Unfortunately, we could not perform this by the competition deadline, and thus we submitted the system that was available at that time, which was based on the BoW (NB-TF.IDF) and character 3-gram features, as shown in row 3 in Table 1 . Our system achieved $72.9 \%$ accuracy on the test by-Article data, ranking $20^{\text {th }} / 42$, and $60.8 \%$ accuracy on the test by-Publisher data, ranking $15^{\text {th }} / 42$.

\section{Conclusion}

We presented our submission to SemEval-2019 Task 4 on Hyperpartisan News Detection. We trained a logistic regression model with a feature set that included word and character $n$-grams, weighted using TF.IDF, after scaling using Naive Bayes. Our system achieved accuracy of $72.9 \%$ and $60.8 \%$ on the test datasets that were labeled by-Article and by-Publisher, respectively.

We further experimented with additional features that represent different aspects of the article's text such as its vocabulary richness, the kind of language it uses according to different lexicons, and its level of complexity. Initial experiments showed that these features hurt the model.

However, with proper pre-processing and scaling, we were able to achieve significant performance gains of up to $2 \%$ absolute in terms of accuracy. Unfortunately, we only obtained these results after the competition's deadline, and thus they were not considered as part of our submission. Yet, we have described them in order to facilitate further research.

\section{Acknowledgments}

This research is part of the Tanbih project, ${ }^{3}$ which aims to limit the effect of "fake news", propaganda and media bias by making users aware of what they are reading. The project is developed in collaboration between the Qatar Computing Research Institute (QCRI), HBKU and the MIT Computer Science and Artificial Intelligence Laboratory (CSAIL).

\footnotetext{
${ }^{3}$ http://tanbih.qcri.org/
} 


\section{References}

Sean Baird, Doug Sibley, and Yuxi Pan. 2017. Talos targets disinformation with fake news challenge victory. https: //blog.talosintelligence.com/2017/ $06 /$ talos-fake-news-challenge.html.

Ramy Baly, Georgi Karadzhov, Dimitar Alexandrov, James Glass, and Preslav Nakov. 2018a. Predicting factuality of reporting and bias of news media sources. In Proceedings of the 2018 Conference on Empirical Methods in Natural Language Processing, EMNLP'18, pages 3528-3539, Brussels, Belgium.

Ramy Baly, Georgi Karadzhov, Abdelrhman Saleh, James Glass, and Preslav Nakov. 2019. Multi-task ordinal regression for jointly predicting the trustworthiness and the leading political ideology of news media. In Proceedings of the 17th Annual Conference of the North American Chapter of the Association for Computational Linguistics: Human Language Technologies, NAACL-HLT '19, Minneapolis, MN, USA.

Ramy Baly, Mitra Mohtarami, James Glass, Lluís Màrquez, Alessandro Moschitti, and Preslav Nakov. 2018b. Integrating stance detection and fact checking in a unified corpus. In Proceedings of the 2018 Conference of the North American Chapter of the Association for Computational Linguistics: Human Language Technologies, NAACL-HLT '18, pages 21-27, New Orleans, LA, USA.

Alberto Barrón-Cedeño, Giovanni Da San Martino, Israa Jaradat, and Preslav Nakov. 2019. Proppy: Organizing news coverage on the basis of their propagandistic content. Information Processing and Management.

Alberto Barrón-Cedeño, Giovanni Da San Martino, Israa Jaradat, and Preslav Nakov. 2019. Proppy: A system to unmask propaganda in online news. In Proceedings of the Thirty-Third AAAI Conference on Artificial Intelligence, AAAI'19, Honolulu, HI, USA.

Ann M Brill. 2001. Online journalists embrace new marketing function. Newspaper Research Journal, 22(2):28.

Carlos Castillo, Marcelo Mendoza, and Barbara Poblete. 2011. Information credibility on Twitter. In Proceedings of the 20th International Conference on the World Wide Web, WWW'11, pages 675-684, Hyderabad, India.

Jacques Ellul. 1965. Propaganda: The Formation of Men's Attitudes. Vintage Books, United States.

Howard Finberg, Martha L Stone, and Diane Lynch. 2002. Digital journalism credibility study. Online News Association. Retrieved November, 3:2003.

Robert Gunning. 1968. The Technique of Clear Writing. McGraw-Hill.
Andreas Hanselowski, Avinesh PVS, Benjamin Schiller, Felix Caspelherr, Debanjan Chaudhuri, Christian M. Meyer, and Iryna Gurevych. 2018. A retrospective analysis of the fake news challenge stance-detection task. In Proceedings of the 27th International Conference on Computational Linguistics, COLING '18, pages 1859-1874, Santa Fe, NM, USA.

Anthony Honore. 1979. Some Simple Measures of Richness of Vocabulary. Association for Literary and Linguistic Computing Bulletin, 7(2):172-177.

Joan B. Hooper. 1975. On assertive predicates. In J. Kimball, editor, Syntax and Semantics, volume 4, page 91124. Academic Press, New York.

Benjamin D Horne and Sibel Adal. 2017. This just in: Fake news packs a lot in title, uses simpler, repetitive content in text body, more similar to satire than real news. In Proceedings of the International Workshop on News and Public Opinion at ICWSM, Montreal, Canada.

Ken Hyland. 2015. The International Encyclopedia of Language and Social Interaction, chapter Metadiscourse. American Cancer Society.

Johannes Kiesel, Maria Mestre, Rishabh Shukla, Emmanuel Vincent, David Corney, Payam Adineh, Benno Stein, and Martin Potthast. 2019. SemEval2019 Task 4: Hyperpartisan news detection. In Proceedings of the 13th International Workshop on Semantic Evaluation, SemEval '19, Minneapolis, MN, USA.

J Peter Kincaid, Robert P Fishburne Jr, Richard L Rogers, and Brad S Chissom. 1975. Derivation of new readability formulas (automated readability index, fog count and flesch reading ease formula) for navy enlisted personnel. Memphis TN Naval Air Station, Research B.

Moshe Koppel, Jonathan Schler, and Elisheva Bonchek-Dokow. 2007. Measuring differentiability: Unmasking pseudonymous authors. Journal of Machine Learning Research, 8:1261-1276.

Vivek Kulkarni, Junting Ye, Steve Skiena, and William Yang Wang. 2018. Multi-view models for political ideology detection of news articles. In Proceedings of the 2018 Conference on Empirical Methods in Natural Language Processing, EMNLP' 18, pages 3518-3527, Brussels, Belgium.

David MJ Lazer, Matthew A Baum, Yochai Benkler, Adam J Berinsky, Kelly M Greenhill, Filippo Menczer, Miriam J Metzger, Brendan Nyhan, Gordon Pennycook, David Rothschild, et al. 2018. The science of fake news. Science, 359(6380):10941096.

Todor Mihaylov, Tsvetomila Mihaylova, Preslav Nakov, Lluís Màrquez, Georgi Georgiev, and Ivan Koychev. 2018. The dark side of news community forums: Opinion manipulation trolls. Internet Research, 28(5):1292-1312. 
Mitra Mohtarami, Ramy Baly, James Glass, Preslav Nakov, Lluís Màrquez, and Alessandro Moschitti. 2018. Automatic stance detection using end-toend memory networks. In Proceedings of the 16th Annual Conference of the North American Chapter of the Association for Computational Linguistics: Human Language Technologies, NAACL-HLT '18, pages 767-776, New Orleans, LA, USA.

Preslav Nakov, Lluís Màrquez, Alberto BarrónCedeño, Pepa Gencheva, Georgi Karadzhov, Tsvetomila Mihaylova, Mitra Mohtarami, and James Glass. 2019. Automatic fact checking using context and discourse information. ACM Journal of Data and Information Quality.

James W Pennebaker, Martha E Francis, and Roger J Booth. 2001. Linguistic inquiry and word count: Liwc 2001. LIWC Operators Manual 2001.

Martin Potthast, Johannes Kiesel, Kevin Reinartz, Janek Bevendorff, and Benno Stein. 2018. A stylometric inquiry into hyperpartisan and fake news. In Proceedings of the 56th Annual Meeting of the Association for Computational Linguistics, ACL '18, pages 231-240, Melbourne, Australia.

Hannah Rashkin, Eunsol Choi, Jin Yea Jang, Svitlana Volkova, and Yejin Choi. 2017. Truth of varying shades: Analyzing language in fake news and political fact-checking. In Proceedings of the 2017 Conference on Empirical Methods in Natural Language Processing, EMNLP'17, pages 2931-2937, Copenhagen, Denmark.

Mark Schmidt, Nicolas Le Roux, and Francis Bach. 2017. Minimizing finite sums with the stochastic average gradient. Mathematical Programming, 162(12):83-112.

Efstathios Stamatatos. 2009. A Survey of Modern Authorship Attribution Methods. Journal of the American Society for Information Science and Technology, 60(3):538-556.

James Thorne and Andreas Vlachos. 2018. Automated fact checking: Task formulations, methods and future directions. In Proceedings of the 27th International Conference on Computational Linguistics, COLING '18, pages 3346-3359, Santa Fe, NM, USA.

Peter D. Turney. 2002. Thumbs up or thumbs down?: Semantic orientation applied to unsupervised classification of reviews. In Proceedings of the 40th Annual Meeting on Association for Computational Linguistics, ACL '02, pages 417-424, Philadelphia, Pennsylvania.

Soroush Vosoughi, Deb Roy, and Sinan Aral. 2018. The spread of true and false news online. Science, 359(6380):1146-1151.

Sida Wang and Christopher D Manning. 2012. Baselines and bigrams: Simple, good sentiment and topic classification. In Proceedings of the 50th Annual
Meeting of the Association for Computational Linguistics, ACL '12, pages 90-94, Jeju Island, Korea.

Theresa Wilson, Janyce Wiebe, and Paul Hoffmann. 2005. Recognizing contextual polarity in phraselevel sentiment analysis. In Proceedings of the Conference on Human Language Technology and Empirical Methods in Natural Language Processing, HLT-EMNLP '05, pages 347-354, Vancouver, Canada.

George Udny Yule. 1944. The Statistical Study of Literary Vocabulary. Cambridge University Press. 